\title{
Standardized Compliance Matrices for General Anisotropic Materials and a Simple Measure of Anisotropy Degree Based on Shear-Extension Coupling Coefficient
}

\author{
Jia-Min Zhao*, Xiao-Xiong Song ${ }^{\dagger}$ and Bin Liu ${ }^{\ddagger}$ \\ $A M L$, Department of Engineering Mechanics \\ Tsinghua University, Beijing 100084 \\ P. R. China \\ Center for Nano and Micro Mechanics \\ Tsinghua University, Beijing 100084 \\ P. R. China \\ *zhaojm12@mails.tsinghua.edu.cn \\ †song-xx12@mails.tsinghua.edu.cn \\ ${ }_{\ddagger}^{\ddagger}$ liubin@tsinghua.edu.cn \\ Received 24 March 2016 \\ Revised 12 June 2016 \\ Accepted 18 June 2016 \\ Published 10 November 2016
}

\begin{abstract}
The compliance matrix for a general anisotropic material is usually expressed in an arbitrarily chosen coordinate system, which brings some confusion or inconvenience in identifying independent elastic material constants and comparing elastic properties between different materials. In this paper, a unique stiffest orientation-based standardized compliance matrix is established, and 18 independent elastic material constants are clearly shown. During the searching process for the stiffest orientation, it is interesting to find from our theoretical analysis and an example that a material with isotropic tensile stiffness does not definitely possess isotropic elasticity. Therefore, the ratio between the maximum and minimum tensile stiffnesses, although widely used, is not a correct measure of anisotropy degree. Alternatively, a simple and correct measure of anisotropy degree based on the maximum shear-extension coupling coefficient in all orientations is proposed. However, for a two-dimensional constitutive relation, both the stiffness ratio and the shear-extension coupling coefficient can be adopted as proper measures of anisotropy degree.
\end{abstract}

Keywords: Anisotropic elasticity; standardized compliance matrix; elastic constants; anisotropic degree.

\footnotetext{
$\ddagger$ Corresponding author.
}

This is an Open Access article published by World Scientific Publishing Company. It is distributed under the terms of the Creative Commons Attribution 4.0 (CC-BY) License. Further distribution of this work is permitted, provided the original work is properly cited. 


\section{Introduction}

The generalized Hooke's law relating stresses to strains for anisotropic elastic materials can be written as

$$
\left[\begin{array}{c}
\varepsilon_{11} \\
\varepsilon_{22} \\
\varepsilon_{33} \\
\gamma_{23} \\
\gamma_{13} \\
\gamma_{12}
\end{array}\right]=\left[\begin{array}{c}
\varepsilon_{11} \\
\varepsilon_{22} \\
\varepsilon_{33} \\
2 \varepsilon_{23} \\
2 \varepsilon_{13} \\
2 \varepsilon_{12}
\end{array}\right]=\left[\begin{array}{llllll}
S_{11} & S_{12} & S_{13} & S_{14} & S_{15} & S_{16} \\
S_{21} & S_{22} & S_{23} & S_{24} & S_{25} & S_{26} \\
S_{31} & S_{32} & S_{33} & S_{34} & S_{35} & S_{36} \\
S_{41} & S_{42} & S_{43} & S_{44} & S_{45} & S_{46} \\
S_{51} & S_{52} & S_{53} & S_{54} & S_{55} & S_{56} \\
S_{61} & S_{62} & S_{63} & S_{64} & S_{65} & S_{66}
\end{array}\right]\left[\begin{array}{c}
\sigma_{11} \\
\sigma_{22} \\
\sigma_{33} \\
\sigma_{23} \\
\sigma_{13} \\
\sigma_{12}
\end{array}\right],
$$

where $\sigma_{i j}, \varepsilon_{i j}$ and $S_{i j}$ are stress, strain and compliance matrix components, respectively. The compliance matrix $\mathbf{S}$ is a symmetric matrix, i.e., $S_{i j}=S_{j i}$. In many textbooks [Ting, 1996; Kaw, 1997; Jones, 1999; Bower, 2011], it is written that a general anisotropic material has 21 independent elastic constants in a compliance matrix. If a material possesses orientational symmetries, the number of independent elastic constants will reduce. For example, an orthotropic material, with three orthogonal planes of material symmetry, has only nine independent elastic constants. In these textbooks, the compliance matrix of orthotropic material is written as

$$
\mathbf{S}^{\text {ortho }}=\left[\begin{array}{cccccc}
S_{11} & S_{12} & S_{13} & 0 & 0 & 0 \\
S_{12} & S_{22} & S_{23} & 0 & 0 & 0 \\
S_{13} & S_{23} & S_{33} & 0 & 0 & 0 \\
0 & 0 & 0 & S_{44} & 0 & 0 \\
0 & 0 & 0 & 0 & S_{55} & 0 \\
0 & 0 & 0 & 0 & 0 & S_{66}
\end{array}\right] .
$$

When we observe these two compliance matrices [Eqs. (1.1) and (1.2)], we find that there is some inconsistence between them. The compliance matrix for orthotropic elastic material is usually expressed in a special coordinate system accounting for the intrinsic orientational symmetry, but the compliance matrix for general anisotropic elastic materials is expressed in an arbitrarily chosen coordinate system. The compliance matrix varies with different coordinate systems. If there is a standardized coordinate system without orientational arbitrariness, as in an orthotropic material, the compliance matrix becomes a standardized one, which provides convenience in comparing the elastic properties among different materials. This standardized compliance matrix can also clearly reveal the number of independent elastic material constants, e.g., 9 for orthotropic materials. Hence, such a straightforward and easy-use standardized compliance matrix for general anisotropic elastic materials is needed and should be included in textbooks or handbooks for students and engineers. 
The number of elastic constants has been a basic problem for a long time. The 21 independent elastic constants of anisotropic material are widely accepted after Green introduced the concept of strain energy and Lord Kelvin proved the existence of Green's strain energy function [Herakovich, 2012]. However, Fedorov [1968] showed that a definite choice of coordinate system may impose three conditions on elastic constants of anisotropic material, resulting in that the number of independent constants cannot exceed 18. Some other researchers also demonstrated the situation of 18 independent constants. Based on the theory of existence of longitudinal wave by Truesdell [1966, 1968], Boulanger and Hayes [1995] discovered two components will vanish when the coordinate axis is the direction making young's modulus reach an extremum value. The same phenomena occurred when the coordinate axis was set as the direction making the axial elastic coefficient extremum by Cowin and Mehrabadi [1995]. They also found the third vanishing component through a specific rotation of coordinate system. It has also been pointed out that the 21 independent elastic constants are consisted of 6 Kelvin moduli, 12 stiffness distributors and 3 orientation angles [Cowin and Mehrabadi, 1992; Rychlewski, 1995; Dellinger et al., 1998; Kowalczyk-Gajewska and Ostrowska-Maciejewska, 2009]. Hence, 18 independent elastic constants are proved, which however has not been clearly presented to students through most textbooks due to the involvement of tensor computation.

In this paper, we propose a unique standardized compliance matrix for general anisotropic materials to show the elastic properties, such as independent elastic constants, in a unified way to avoid confusion. The paper is structured as follows. In Sec. 2, we propose a unique stiffest orientation-based standardized compliance matrix, and compare it with the symmetry orientation-based standardized compliance matrix. We investigate the relation between the symmetry of tensile stiffness and the symmetry of the elastic properties in Sec. 3. By interestingly noting that the ratio between the maximum and minimum tensile stiffnesses is not a correct measure of anisotropy degree, we propose a simple and correct measure of anisotropy degree based on the maximum shear-extension coupling coefficient in Sec. 4. Conclusions are summarized in Sec. 5 .

\section{The Definition and Characteristics of a Standardized Compliance Matrix for General Anisotropic Materials}

\subsection{The definition of the stiffest orientation-based standardized compliance matrix}

To exclude the orientational arbitrariness of a coordinate system and obtain a unique standardized compliance matrix, a special coordinate system based on the material intrinsic orientation is needed. For orthotropic materials, the orientational symmetry can be adopted in choosing the coordinate system. For general anisotropic materials, however, there is no orientational symmetry. To deal with this situation, 
we propose that the material intrinsic orientation with extreme stiffness is used in establishing a standardized coordinate system $\hat{x}_{1}-\hat{x}_{2}-\hat{x}_{3}$, and the specific rules or conditions are suggested as follows.

Condition I: The $\hat{x}_{1}$-axis makes $S_{11}$ reach the global minimum, i.e., the stiffest direction in the whole orientations.

Condition II: The $\hat{x}_{2}$-axis makes $S_{22}$ global minimum within the plane perpendicular to $\hat{x}_{1}$-axis.

Condition III: The $\hat{x}_{3}$-axis complies with the right-hand coordinate system rule.

Condition IV: $S_{14}$ and $S_{25}$ reach their minimum values under above conditions.

The coordinate system $\hat{x}_{1}-\hat{x}_{2}-\hat{x}_{3}$ satisfying Conditions I-IV is named as the stiffest orientation-based standardized coordinate system, or STF standardized coordinate system for abbreviation. The corresponding compliance matrix in this coordinate system is named as the stiffest orientation-based standardized compliance matrix, or STF standardized compliance matrix for abbreviation, and denoted as $\hat{\mathbf{S}}$. The first three conditions are obvious. For a general anisotropic material, as shown in Fig. 1, there are four coordinate systems satisfying Conditions I-III, since one stiffest direction has two options for $\hat{x}_{1}$-axis, or $\hat{x}_{2}$-axis. The relations among these four candidate coordinate systems (a)-(d) are also shown in Fig. 1. Rotating Coordinate system (a) $\pi$ angle around $\hat{x}_{1}^{a}$-axis yields Coordinate system (b). Rotating Coordinate system (a) $\pi$ angle around $\hat{x}_{2}^{a}$ axis yields Coordinate system (c). Rotating Coordinate system (a) $\pi$ angle around $\hat{x}_{3}^{a}$-axis yields Coordinate system $(\mathrm{d})$. The compliance matrices under coordinate

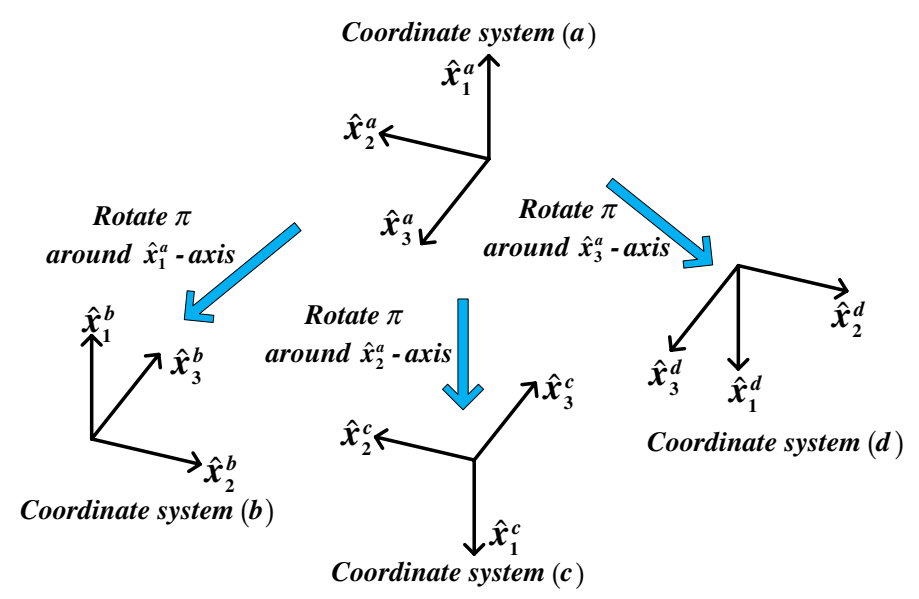

Fig. 1. The four coordinate systems satisfying Conditions I-III. 
systems (a)-(d) are denoted as $\mathbf{S}^{a}, \mathbf{S}^{b}, \mathbf{S}^{c}$ and $\mathbf{S}^{d}$, respectively. Supposing

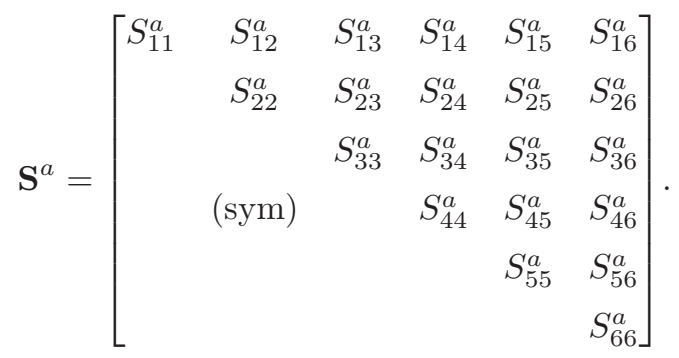

According to transformation relation among the four coordinate systems (see Appendix A), $\mathbf{S}^{b}, \mathbf{S}^{c}$ and $\mathbf{S}^{d}$ can be represented by the components of $\mathbf{S}^{a}$ as follows

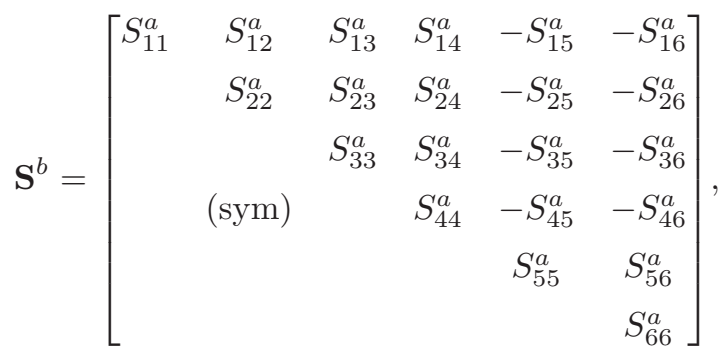

$$
\begin{aligned}
& \mathbf{S}^{c}=\left[\begin{array}{cccccc}
S_{11}^{a} & S_{12}^{a} & S_{13}^{a} & -S_{14}^{a} & S_{15}^{a} & -S_{16}^{a} \\
& S_{22}^{a} & S_{23}^{a} & -S_{24}^{a} & S_{25}^{a} & -S_{26}^{a} \\
& & S_{33}^{a} & -S_{34}^{a} & S_{35}^{a} & -S_{36}^{a} \\
& & & & & \\
& & & & & S_{55}^{a} \\
& & & & & -S_{56}^{a} \\
& & & & & S_{66}^{a}
\end{array}\right], \\
& \mathbf{S}^{d}=\left[\begin{array}{cccccc}
S_{11}^{a} & S_{12}^{a} & S_{13}^{a} & -S_{14}^{a} & -S_{15}^{a} & S_{16}^{a} \\
& S_{22}^{a} & S_{23}^{a} & -S_{24}^{a} & -S_{25}^{a} & S_{26}^{a} \\
& & S_{33}^{a} & -S_{34}^{a} & -S_{35}^{a} & S_{36}^{a} \\
& & & & & \\
& & & & S_{45}^{a} & -S_{46}^{a} \\
& & & & & \\
& & & & & S_{56}^{a}
\end{array}\right] .
\end{aligned}
$$

Obviously, the compliance matrices under these four coordinate systems are different. Therefore, we propose that the unique standardized compliance matrix is the one with minimal $S_{14}$ and $S_{25}$ among these four matrices, i.e., Condition IV.

Conditions I-IV can ensure the uniqueness of this standardized compliance matrix for a general anisotropic material. However, for some materials with special symmetries, the uniqueness of STF standardized compliance matrix may not 


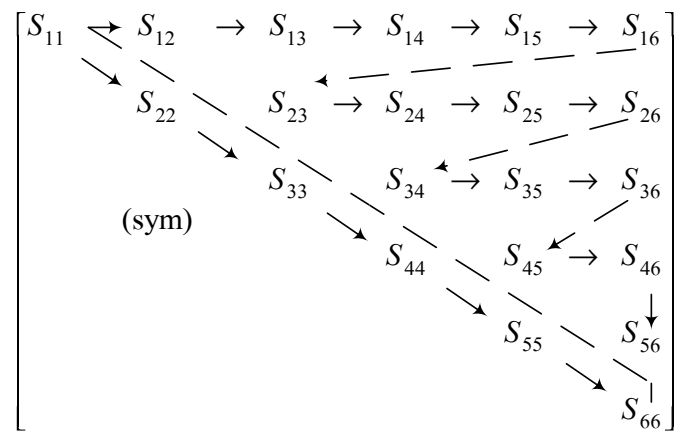

Fig. 2. The specified sequence of compliance matrix components.

be guaranteed by Conditions I-IV. Therefore, a more general rule in determining a unique standardized compliance matrix is proposed as follows. Firstly, a specified sequence of compliance matrix components is introduced as

$$
\begin{array}{r}
\left(S_{11}, S_{22}, S_{33}, S_{44}, S_{55}, S_{66}, S_{12}, S_{13}, S_{14}, S_{15}, S_{16},\right. \\
\left.S_{23}, S_{24}, S_{25}, S_{26}, S_{34}, S_{35}, S_{36}, S_{45}, S_{46}, S_{56}\right),
\end{array}
$$

and is schematically shown in Fig. 2. Similar to Conditions I-IV, we first find coordinate systems to make $S_{11}$ global minimum. For the rest components in the sequence, we then sequently search for their global minimums while keeping the previous components unchanged. It should be pointed out that for most situations, Conditions I-IV are consistent with this general rule, and are relatively easier to use.

For any anisotropic material, adopting such unique STF standardized compliance matrix can eliminate the arbitrariness brought from different coordinate systems, and make it convenient to compare the elastic properties between different materials. For a general anisotropic material, the unique STF standardized compliance matrix corresponds to the unique STF standardized coordinate system. However, for materials with some symmetry, the STF standardized coordinate system might not be unique. More discussion about this situation will be given in Sec. 3.1.

\subsection{The characteristic of the stiffest orientation-based standardized compliance matrix for general anisotropic materials}

For general anisotropic materials, we will illustrate and prove that there are three zeroes in the upper triangle of the standardized compliance matrix.

We adopt an example to understand the origin of these zero compliance components. Figure 3 shows an initially rectangular representative volume element (RVE) subject to a uniaxial tensile stress $\sigma_{11}$ along the $\hat{x}_{1}$-direction. In the following, we 


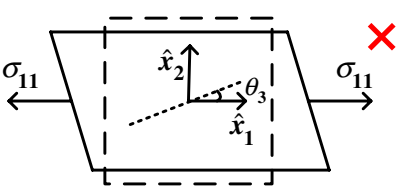

(a)

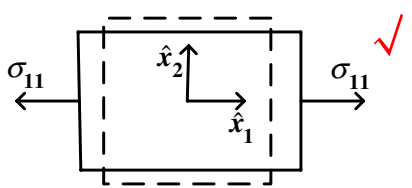

(b)

Fig. 3. The deformation of RVE subject to a uniaxial tensile stress $\sigma_{11}$ along the $\hat{x}_{1}$-direction: (a) deformed RVE in parallelogram shape and (b) deformed RVE in rectangle shape.

use proof by contradiction to demonstrate that the deformed RVE is still a rectangle. Firstly, we assume that the deformed RVE under $\sigma_{11}$ becomes a parallelogram as shown in Fig. 3(a), and we can note that the direction rotating anticlockwise slightly from $\hat{x}_{1}$-direction (denoted as a dotted line) is less stretchable and therefore has a larger tensile stiffness, which is contradictory to the condition that the global maximum stiffness is achieved in $\hat{x}_{1}$-direction. Hence the deformed RVE must be a rectangle as shown in Fig. $3(\mathrm{~b})$, which implies that the $\sigma_{11}$ along the $\hat{x}_{1}$-direction cannot lead to the shear strain $\gamma_{12}$, i.e., $\hat{S}_{16}=0$. Similarly, $\hat{S}_{15}=0$ and $\hat{S}_{24}=0$ can be obtained. These conclusions can also be proved strictly in an analytical way.

Compliance components vary with the rotation of the coordinate system. When rotating the STF standardized coordinate system $\hat{x}_{1}-\hat{x}_{2}-\hat{x}_{3}$ around $\hat{x}_{3}$-axis through an angle $\theta_{3}$ (in this paper all rotations follow the right-hand rule), the compliance component $S_{11}$ can be expressed as

$$
\begin{aligned}
S_{11}\left(\hat{S}_{i j}, \theta_{3}\right)= & \cos ^{4}\left(\theta_{3}\right) \hat{S}_{11}+\sin ^{4}\left(\theta_{3}\right) \hat{S}_{22}+\sin ^{2}\left(\theta_{3}\right) \cos ^{2}\left(\theta_{3}\right)\left(\hat{S}_{66}+2 \hat{S}_{12}\right) \\
& +2 \sin \left(\theta_{3}\right) \cos ^{3}\left(\theta_{3}\right) \hat{S}_{16}+2 \sin ^{3}\left(\theta_{3}\right) \cos \left(\theta_{3}\right) \hat{S}_{26}
\end{aligned}
$$

According to Condition I and the above equation, $\hat{S}_{11}$ is the global minimum of $S_{11}$, and is achieved along $\hat{x}_{1}$-axis, i.e.,

$$
\left.\frac{\partial S_{11}}{\partial \theta_{3}}\right|_{\theta_{3}=0}=2 \hat{S}_{16}=0 .
$$

Similarly,

$$
\left.\frac{\partial S_{11}}{\partial \theta_{2}}\right|_{\theta_{2}=0}=-2 \hat{S}_{15}=0 .
$$

According to Condition II, $\hat{S}_{22}$ is the minimum of $S_{22}$ in the plane perpendicular to $\hat{x}_{1}$-axis, and is achieved along $\hat{x}_{2}$-axis, i.e.,

$$
\left.\frac{\partial S_{22}}{\partial \theta_{1}}\right|_{\theta_{1}=0}=2 \hat{S}_{24}=0 .
$$


Based on the above discussions, the final form of the STF standardized compliance matrix for general anisotropic materials is

$$
\hat{\mathbf{S}}=\left[\begin{array}{cccccc}
\hat{S}_{11} & \hat{S}_{12} & \hat{S}_{13} & \hat{S}_{14} & 0 & 0 \\
& \hat{S}_{22} & \hat{S}_{23} & 0 & \hat{S}_{25} & \hat{S}_{26} \\
& & \hat{S}_{33} & \hat{S}_{34} & \hat{S}_{35} & \hat{S}_{36} \\
& (\mathrm{sym}) & & \hat{S}_{44} & \hat{S}_{45} & \hat{S}_{46} \\
& & & & \hat{S}_{55} & \hat{S}_{56} \\
& & & & & \hat{S}_{66}
\end{array}\right] .
$$

Obviously, there are only 18 nonzero components in the upper triangle of the standardized compliance matrix, which can be naturally selected as 18 independent elastic material constants. Here, we intentionally use "elastic material constants" to reflect their material intrinsic feature, which is independent of the coordinate orientation. Therefore, 18 independent elastic material constants together with three arbitrariness of the coordinate orientation result in 21 independent elastic constants in the usual form of the compliance matrix for general anisotropic materials. We suggest including these statements in the textbooks to avoid possible confusions.

It should be mentioned that there are many choices for these 18 independent elastic material constants. For example, some researchers [Cowin and Mehrabadi, 1992; Rychlewski, 1995; Dellinger et al., 1998; Kowalczyk-Gajewska and OstrowskaMaciejewska, 2009] had pointed out that the 21 independent elastic constants in Kelvin notation are consisted of 6 Kelvin moduli, 12 stiffness distributors and 3 orientation angles. The 6 Kelvin moduli are the eigenvalues of the compliance matrix and do not vary with the coordinate system. The advantage of using the group of independent elastic material constants from Kelvin notation is the reflection of mathematical essences of an elastic constitutive tensor, such as eigenvalues and tensor invariants. However, the independent elastic material constants based on the components of a standardized compliance matrix proposed in this paper have more straightforward physical meaning, since each constant represents the strain-stress ratio under uniaxial loading and it is obtained directly and easily in experiments [Cazzani and Rovati, 2003; Rovati and Taliercio, 2003]. We therefore recommend using the proposed elastic material constants, just like the Young's modulus and Poisson ratio for isotropic materials are widely used, but they are not elastic material constants from Kelvin notation.

\subsection{The number of independent elastic material constants for symmetric materials and two-dimensional materials}

When a material has some orientational symmetry, more material intrinsic directions can be used to set up a standardized coordinate system without the arbitrariness of the coordinate orientation. As demonstrated above, only under a 
standardized coordinate system, the number of independent elastic material constants can be correctly exhibited.

For a material with a single symmetry plane (or monoclinic symmetry), if $x_{1}$ axis is taken as the normal direction of the symmetric plane, and $x_{2}$-axis is taken as the stiffest direction in the plane, a standardized coordinate system is then set up. The corresponding standardized compliance matrix is

$$
\mathbf{S}^{\mathrm{sym}}=\left[\begin{array}{cccccc}
S_{11}^{\mathrm{sym}} & S_{12}^{\mathrm{sym}} & S_{13}^{\mathrm{sym}} & S_{14}^{\mathrm{sym}} & 0 & 0 \\
& S_{22}^{\mathrm{sym}} & S_{23}^{\mathrm{sym}} & 0 & 0 & 0 \\
& & S_{33}^{\mathrm{sym}} & S_{34}^{\mathrm{sym}} & 0 & 0 \\
& (\mathrm{sym}) & & S_{44}^{\mathrm{sym}} & 0 & 0 \\
& & & & S_{55}^{\mathrm{sym}} & S_{56}^{\mathrm{sym}} \\
& & & & & S_{66}^{\text {sym }}
\end{array}\right] .
$$

The superscript "sym" implies that the material orientational symmetry is used in selecting the coordinate system, and the corresponding compliance matrix is called as the symmetry orientation-based standardized compliance matrix, or SYM standardized compliance matrix for abbreviation. In the above equation $S_{24}^{\mathrm{sym}}=0$ has been used according to Eq. (2.8), and noting that $S_{22}^{\mathrm{sym}}$ is the minimum of $S_{22}$ in the symmetry plane. It is noted from Eq. (2.10) that a material with a single symmetry plane has only 12 independent elastic material constants, different from the statement in the textbooks that there are 13 independent elastic constants, since the arbitrariness of the coordinate orientation is usually not emphasized. Cowin [1995] also proved that the appropriate selection of the coordinate system may reduce the number of the distinct constants for monoclinic symmetry by one.

For an orthotropic material with three orthogonal symmetry planes, $x_{1}$-axis and $x_{2}$-axis are usually taken as two normal directions of the orthogonal symmetry planes, and the three arbitrariness of the coordinate orientation are naturally excluded. The corresponding SYM standardized compliance matrix equation (1.2) can correctly exhibit nine independent elastic material constants, as presented in the textbooks.

When only the two-dimensional anisotropic elasticity is under consideration, the sub-compliance-matrix $\mathbf{S}_{2 D}$ has six components, i.e., $S_{11}, S_{22}, S_{66}, S_{12}, S_{16}$ and $S_{26}$. The corresponding STF standardized compliance matrix is easily obtained as

$$
\hat{\mathbf{S}}_{2 D}=\left[\begin{array}{ccc}
\hat{S}_{11} & \hat{S}_{12} & 0 \\
\hat{S}_{12} & \hat{S}_{22} & \hat{S}_{26} \\
0 & \hat{S}_{26} & \hat{S}_{66}
\end{array}\right] .
$$

This standardized compliance matrix clearly reveals that there are only five independent elastic material constants, not six. 


\subsection{Comparison between two types of standardized compliance matrices: The stiffest orientation-based one and symmetry orientation-based one}

As mentioned above, two types of material intrinsic directions, i.e., the direction with an extreme stiffness and the one related to some symmetries, can be used to establish a special coordinate system. The corresponding compliance matrix without the arbitrariness of coordinate system orientation is called a standardized one in this paper. If only the stiffest directions are adopted in establishing the special coordinate system, a STF standardized compliance matrix is obtained, while if any direction related to some symmetries is adopted, a SYM standardized compliance matrix is obtained.

The STF standardized compliance matrix and SYM standardized compliance matrix have different advantages and applications. The former exists for any anisotropic material and has a unique expression, which is convenient in comparing among different materials. The latter only exists for materials with orientational symmetry, but can exhibit the number of independent material constants more clearly than the former.

For materials with some symmetry planes, one might speculate that the special coordinate system based on the extreme stiffness direction coincides with the one based on the normal directions of the symmetry planes. This is not always true, and will be demonstrated by the following example.

An overall orthotropic laminate material is composed of unidirectional fiberreinforced laminas, as shown in Fig. 4. The composite laminate has four laminas

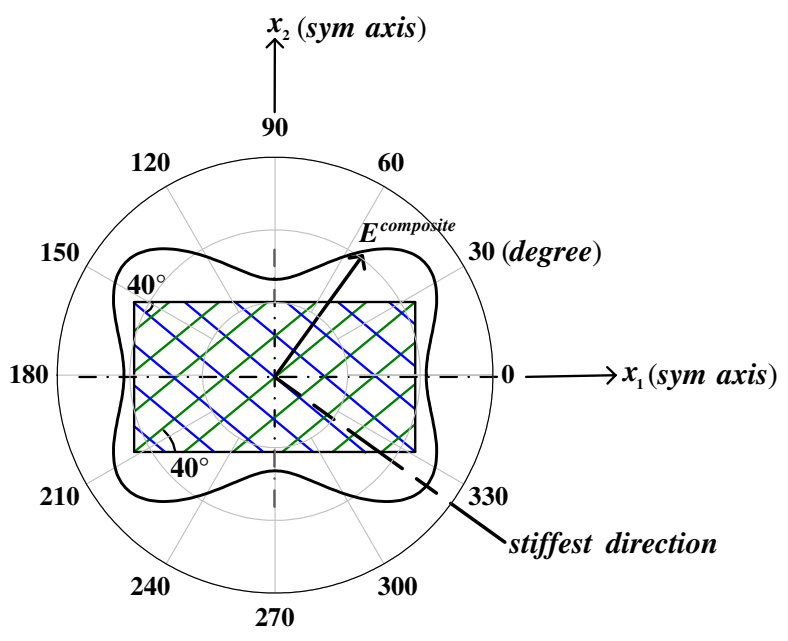

(a)

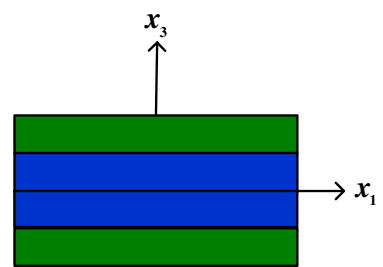

(b)

Fig. 4. The distribution of values of $E^{\text {compostie }}$ for composite laminate in different directions: (a) top view of the composite laminate and (b) side view of the composite laminate. 
with equal thickness and the set of fiber orientations $\left[40^{\circ},-40^{\circ},-40^{\circ}, 40^{\circ}\right]$. The compliance matrix of the unidirectional fiber-reinforced lamina under its local principle coordinate system (aligned with the fiber direction) is supposed as

$$
\mathbf{S}^{\text {lamina }}=\frac{1}{E_{1}^{\text {lamina }}}\left[\begin{array}{ccc}
1 & -0.3 & 0 \\
-0.3 & 100 & 0 \\
0 & 0 & 8
\end{array}\right]
$$

where $E_{1}^{\text {lamina }}$ is the Young's modulus of the fiber-reinforced lamina along the direction of fiber orientation. According to the classical laminate theory, the inplane strains for different laminas are the same, thus the compliance matrix for the laminate can be obtained. The effective Young's modulus in different directions $E^{\text {composite }}$ for composite laminate is shown in Fig. 4(a). It is found that the stiffest direction (or the direction with the maximum $E^{\text {composite }}$ ) is not along any symmetric axis, $x_{1}$-axis or $x_{2}$-axis. Therefore, the STF standardized compliance matrix and the SYM standardized compliance matrix of this composite laminate are different.

\section{Determining Material Orientational Symmetry Through the Searching Process of the Stiffest Orientation-Based Standardized Compliance Matrix}

Materials sometimes do not show their orientational symmetry in their appearance. In this case, we may firstly treat it as a general anisotropic material, and measure its compliance components in an arbitrarily chosen coordinate system. We then search for the STF standardized coordinate system and the corresponding STF standardized compliance matrix. During this searching process, some information, such as the number of STF standardized coordinate systems and a sphere of orientation colored by the values of uniaxial tensile stiffness, can be obtained and may provide hints on the material orientational symmetry, but it is interesting to find that not all these hints can definitely lead to positive results, as discussed later in this section.

\subsection{Symmetry investigation on materials with two stiffest orientation-based standardized coordinate systems}

According to Conditions I-IV in Sec. 2, any material has a unique STF standardized compliance matrix. For general anisotropic materials, this standardized compliance matrix can only be obtained under a single standardized coordinate system. If the STF standardized compliance matrix can be obtained under two STF standardized coordinate systems, we will prove that the material has a symmetry plane.

The two STF standardized coordinate systems are assumed $\hat{x}_{1}^{(1)}-\hat{x}_{2}^{(1)}-\hat{x}_{3}^{(1)}$ and $\hat{x}_{1}^{(2)}-\hat{x}_{2}^{(2)}-\hat{x}_{3}^{(2)}$, under which the STF standardized compliance matrices $\hat{\mathbf{S}}$ are identical. For convenience, a reference coordinate system $X_{1}-X_{2}-X_{3}$, as shown in Fig. 5 , is introduced. In order to show schematics clearly, the origins of different 


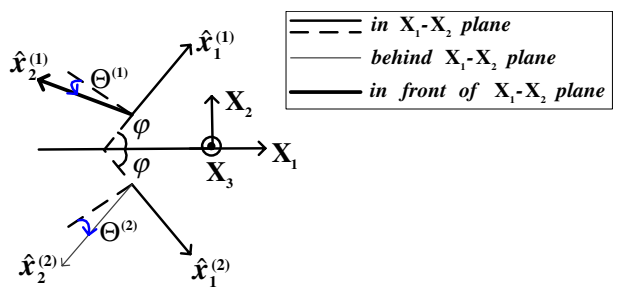

Fig. 5. The two STF standardized coordinate systems with identical STF standardized compliance matrices.

coordinate systems are sometimes translationally shifted in this paper. Without loss of generality, it is assumed that $X_{1}$-axis is the bisector of angle $2 \varphi$ between $\hat{x}_{1}^{(1)}$-axis and $\hat{x}_{1}^{(2)}$-axis, and $X_{2}$-axis is also within the plane of $\hat{x}_{1}^{(1)}$-axis and $\hat{x}_{1}^{(2)}$-axis. The angle around $\hat{x}_{1}^{(1)}$-axis from $X_{1}-X_{2}$ plane to $\hat{x}_{2}^{(1)}$-axis is denoted as $\Theta^{(1)}$, which takes a positive value when following the right-hand rule. The angle around $\hat{x}_{1}^{(2)}$-axis from $X_{1}-X_{2}$ plane to $\hat{x}_{2}^{(2)}$-axis is denoted as $\Theta^{(2)}$.

Because $\hat{x}_{1}^{(1)}-\hat{x}_{2}^{(1)}-\hat{x}_{3}^{(1)}$ and $\hat{x}_{1}^{(2)}-\hat{x}_{2}^{(2)}-\hat{x}_{3}^{(2)}$ are only two STF standardized coordinate systems, it is easy to know that the rotations from the coordinate system $\hat{x}_{1}^{(1)}-\hat{x}_{2}^{(1)}-\hat{x}_{3}^{(1)}$ to $\hat{x}_{1}^{(2)}-\hat{x}_{2}^{(2)}-\hat{x}_{3}^{(2)}$ should be the same as those from the coordinate system $\hat{x}_{1}^{(2)}-\hat{x}_{2}^{(2)}-\hat{x}_{3}^{(2)}$ to $\hat{x}_{1}^{(1)}-\hat{x}_{2}^{(1)}-\hat{x}_{3}^{(1)}$, as shown in Fig. 6 . Therefore $\Theta^{(1)}$ should be equal to $\Theta^{(2)}$ (denoted by $\Theta$ later), and this relation can also been proved analytically as presented in Appendix B.

Through the same rotations (rotating $-\Theta$ around first axis and then rotating $-\varphi$ around the third axis), $\hat{x}_{1}^{(1)}-\hat{x}_{2}^{(1)}-\hat{x}_{3}^{(1)}$ and $\hat{x}_{1}^{(2)}-\hat{x}_{2}^{(2)}-\hat{x}_{3}^{(2)}$ become $X_{1}-X_{2}-X_{3}$ and

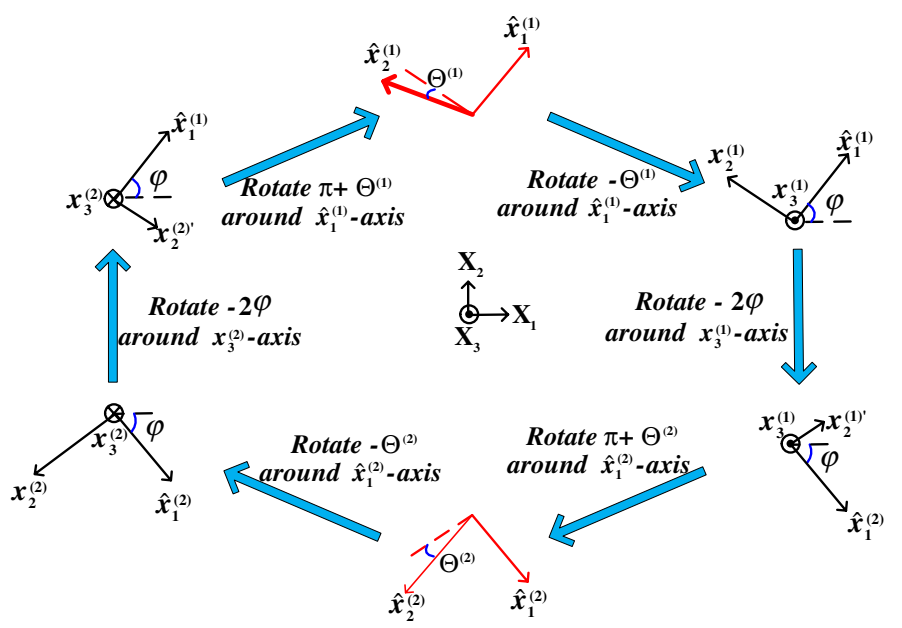

Fig. 6. The rotation relation between coordinate systems $\hat{x}_{1}^{(1)}-\hat{x}_{2}^{(1)}-\hat{x}_{3}^{(1)}$ and $\hat{x}_{1}^{(2)}-\hat{x}_{2}^{(2)}-\hat{x}_{3}^{(2)}$. 


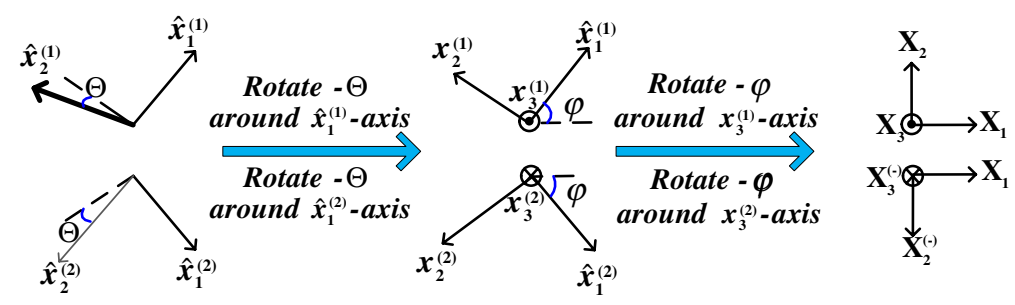

Fig. 7. Schematics of obtaining the coordinate systems through the same rotations from coordinate systems $\hat{x}_{1}^{(1)}-\hat{x}_{2}^{(1)}-\hat{x}_{3}^{(1)}$ and $\hat{x}_{1}^{(2)}-\hat{x}_{2}^{(2)}-\hat{x}_{3}^{(2)}$.

$X_{1}-X_{2}^{(-)}-X_{3}^{(-)}$, respectively, as shown in Fig. 7. Obviously, the compliance matrices under $X_{1}-X_{2}-X_{3}$ and $X_{1}-X_{2}^{(-)}-X_{3}^{(-)}$coordinate systems are the same. It also noted that $X_{1}-X_{2}-X_{3}$ can be obtained by rotating $X_{1}-X_{2}^{(-)}-X_{3}^{(-)} \pi$ around $X_{1}$-axis, which leads eight opposite values in the components for two corresponding compliance matrices, similar to Eqs. (2.1) and (2.2). From these relations, the compliance matrix under $X_{1}-X_{2}-X_{3}$ should therefore have eight zeroes in the upper triangle as,

$$
\mathbf{S}_{X_{1}-X_{2}-X_{3}}=\left[\begin{array}{cccccc}
S_{11}^{(0)} & S_{12}^{(0)} & S_{13}^{(0)} & S_{14}^{(0)} & 0 & 0 \\
& S_{22}^{(0)} & S_{23}^{(0)} & S_{24}^{(0)} & 0 & 0 \\
& & S_{33}^{(0)} & S_{34}^{(0)} & 0 & 0 \\
& & & S_{44}^{(0)} & 0 & 0 \\
& & & & S_{55}^{(0)} & S_{56}^{(0)} \\
& & & & & S_{66}^{(0)}
\end{array}\right]
$$

which implies that $X_{2}-X_{3}$ plane is a symmetry plane for this material and is normal to the angle bisector between $\hat{x}_{1}^{(1)}$-axis and $\hat{x}_{1}^{(2)}$-axis.

\subsection{Identification of the material symmetry through the orientational variation of $S_{11}$}

According to Condition I for a STF standardized coordinate system, determining $\hat{x}_{1}$-axis needs to search for the global minimum $S_{11}$ over all possible orientations. Therefore, a sphere of orientation colored by the values of $S_{11}$ as shown in Fig. 8 can be naturally obtained during the searching process. By observing this sphere, we can find the symmetry of $S_{11}$ if there is any. Our question is that, does the symmetry of $S_{11}$ definitely imply the same symmetry of material elastic properties? In specific, three types of symmetries, i.e., mirror symmetry, transversely isotropic symmetry and isotropic symmetry, are discussed as follows. Euler angles $\alpha, \beta$ and $\gamma$ shown in Fig. $8(\mathrm{a})$ are adopted to describe spatial orientations with respect to a reference coordinate system $X_{1}-X_{2}-X_{3}$. 


\subsubsection{A mirror symmetry in the orientational variation of $S_{11}$}

If a sphere of orientation colored by $S_{11}$ possesses a mirror symmetry, without loss of generality, we assume that $X_{2}-X_{3}$ plane is the symmetry plane, as shown in Fig. 8(b). Obviously, for any $\alpha$ and $\beta$, the direction expressed by Euler angles $(\alpha, \beta)$ and the direction $(\alpha, \pi-\beta)$ are symmetrical about the $X_{2}-X_{3}$ plane, and their corresponding $S_{11}$ are the same, i.e.,

$$
S_{11}\left(\alpha, \beta, S_{i j}^{(0)}\right)=S_{11}\left(\alpha, \pi-\beta, S_{i j}^{(0)}\right),
$$

where $S_{i j}^{(0)}$ are the components of the compliance matrix under $X_{1}-X_{2}-X_{3}$ coordinate system. From the relation between the original compliance matrix and the rotated one (see Appendix A), the detailed expression of Eq. (3.2) can be obtained as

$$
\begin{aligned}
0= & S_{11}\left(\alpha, \beta, S_{i j}^{(0)}\right)-S_{11}\left(\alpha, \pi-\beta, S_{i j}^{(0)}\right) \\
= & -4 \sin (\beta) \cos (\beta)\left\{\cos ^{2}(\beta)\left[\cos (\alpha) S_{15}^{(0)}-\sin (\alpha) S_{16}^{(0)}\right]\right. \\
& +\cos (\alpha) \sin ^{2}(\beta)\left[\sin ^{2}(\alpha)\left(S_{25}^{(0)}+S_{46}^{(0)}\right)+\cos ^{2}(\alpha) S_{35}^{(0)}\right] \\
& \left.-\sin (\alpha) \sin ^{2}(\beta)\left[\sin ^{2}(\alpha) S_{26}^{(0)}+\cos ^{2}(\alpha)\left(S_{36}^{(0)}+S_{45}^{(0)}\right)\right]\right\} .
\end{aligned}
$$

Because the above equation holds for any $\alpha$ and $\beta$, it can be inferred that

$$
S_{15}^{(0)}=S_{16}^{(0)}=S_{26}^{(0)}=S_{35}^{(0)}=S_{25}^{(0)}+S_{46}^{(0)}=S_{36}^{(0)}+S_{45}^{(0)}=0
$$

which are not consistent with the conditions as shown in Eq. (3.1) for the material elastic properties with mirror symmetry about $X_{2}-X_{3}$ plane. This implies that a material with the mirror symmetry of the tensile stiffness does not definitely possess the same symmetry in its elastic properties.

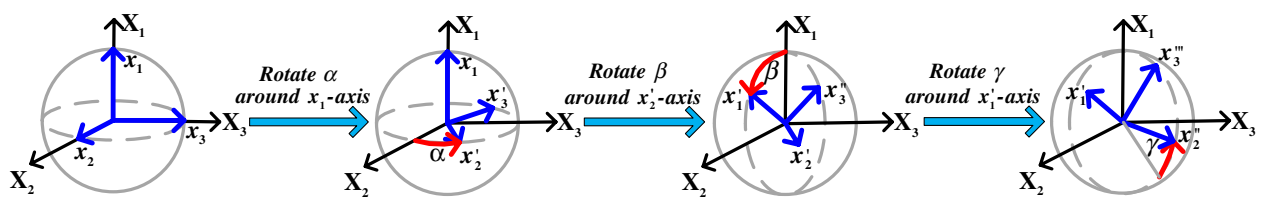

(a)

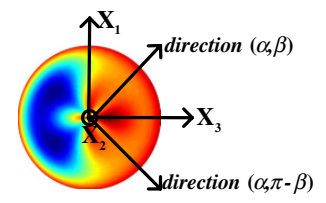

(b)

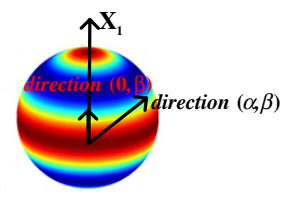

(c)

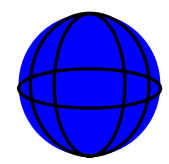

(d)

Fig. 8. Schematics of $S_{11}$ orientational variation with different symmetries. (a) Euler angles $\alpha$, $\beta$ and $\gamma$, (b) mirror symmetry case, (c) transversely isotropic symmetry case and (d) isotropic symmetry case. 


\subsubsection{A transversely isotropic symmetry in the orientational variation of $S_{11}$}

A transversely isotropic symmetry is a much higher symmetry than a mirror symmetry discussed above. If a sphere of orientation colored by $S_{11}$ possesses a transversely isotropic symmetry, without loss of generality, $X_{2}-X_{3}$ plane is assumed to be the transversely isotropic plane, as shown in Fig. 8(c). For any $\alpha$ and $\beta$, the direction expressed by Euler angles $(\alpha, \beta)$ and the direction $(0, \beta)$ have the same $S_{11}$, i.e.,

$$
S_{11}\left(\alpha, \beta, S_{i j}^{(0)}\right)=S_{11}\left(0, \beta, S_{i j}^{(0)}\right) .
$$

From the relation between the original compliance matrix and the rotated one (see Appendix A), the detailed expression of Eq. (3.5) can be obtained as

$$
\begin{aligned}
& 0=S_{11}\left(\alpha, \beta, S_{i j}^{(0)}\right)-S_{11}\left(0, \beta, S_{i j}^{(0)}\right) \\
& =\sin ^{4}(\alpha) \sin ^{4}(\beta) S_{22}^{(0)}+\left[\cos ^{4}(\alpha)-1\right] \sin ^{4}(\beta) S_{33}^{(0)} \\
& +\sin ^{2}(\alpha) \cos ^{2}(\alpha) \sin ^{4}(\beta)\left(S_{44}^{(0)}+2 S_{23}^{(0)}\right) \\
& -\sin ^{2}(\alpha) \sin ^{2}(\beta) \cos ^{2}(\beta)\left[S_{55}^{(0)}-S_{66}^{(0)}-2\left(S_{12}^{(0)}-S_{13}^{(0)}\right)\right] \\
& -2 \sin (\alpha) \cos (\alpha) \sin ^{2}(\beta) \cos ^{2}(\beta)\left(S_{14}^{(0)}+S_{56}^{(0)}\right) \\
& -2 \sin ^{2}(\alpha) \cos (\alpha) \sin ^{3}(\beta) \cos (\beta)\left(S_{25}^{(0)}+S_{46}^{(0)}\right) \\
& +2 \sin (\alpha) \cos ^{2}(\alpha) \sin ^{3}(\beta) \cos (\beta)\left(S_{36}^{(0)}+S_{45}^{(0)}\right) \\
& +2[1-\cos (\alpha)] \sin (\beta) \cos ^{3}(\beta) S_{15}^{(0)}+2 \sin (\alpha) \sin (\beta) \cos ^{3}(\beta) S_{16}^{(0)} \\
& -2 \sin ^{3}(\alpha) \cos (\alpha) \sin ^{4}(\beta) S_{24}^{(0)}+2 \sin ^{3}(\alpha) \sin ^{3}(\beta) \cos (\beta) S_{26}^{(0)} \\
& -2 \sin (\alpha) \cos ^{3}(\alpha) \sin ^{4}(\beta) S_{34}^{(0)}+2\left[1-\cos ^{3}(\alpha)\right] \sin ^{3}(\beta) \cos (\beta) S_{35}^{(0)} \text {. }
\end{aligned}
$$

For any $\alpha$ and $\beta$, the following relations can satisfy Eq. (3.6),

$$
\begin{aligned}
& S_{22}^{(0)}=S_{33}^{(0)}, \quad S_{55}^{(0)}-S_{66}^{(0)}=2\left(S_{12}^{(0)}-S_{13}^{(0)}\right), \quad S_{44}^{(0)}=2\left(S_{22}^{(0)}-S_{23}^{(0)}\right), \\
& S_{14}^{(0)}+S_{56}^{(0)}=S_{25}^{(0)}+S_{46}^{(0)}=S_{36}^{(0)}+S_{45}^{(0)}=0, \\
& S_{15}^{(0)}=S_{16}^{(0)}=S_{24}^{(0)}=S_{26}^{(0)}=S_{34}^{(0)}=S_{35}^{(0)}=0 .
\end{aligned}
$$

However, Eq. (3.7) is not consistent with the following conditions for the transversely isotropic symmetry with the isotropic $X_{2}-X_{3}$ plane on material elastic properties,

$$
\begin{aligned}
& S_{22}^{(0)}=S_{33}^{(0)}, \quad S_{55}^{(0)}=S_{66}^{(0)}, \quad S_{12}^{(0)}=S_{13}^{(0)}, \quad S_{44}^{(0)}=2\left(S_{22}^{(0)}-S_{23}^{(0)}\right), \\
& S_{14}^{(0)}=S_{56}^{(0)}=S_{25}^{(0)}=S_{46}^{(0)}=S_{36}^{(0)}=S_{45}^{(0)}=0, \\
& S_{15}^{(0)}=S_{16}^{(0)}=S_{24}^{(0)}=S_{26}^{(0)}=S_{34}^{(0)}=S_{35}^{(0)}=0 .
\end{aligned}
$$


This also implies that a material with the transversely isotropic symmetry of the tensile stiffness does not definitely possess the same symmetry in its elastic properties.

\subsubsection{An isotropic symmetry in the orientational variation of $S_{11}$}

An isotropic symmetry is the highest symmetry. If a sphere of orientation colored by $S_{11}$ possesses an isotropic symmetry, for any $\alpha$ and $\beta$, the direction expressed by Euler angles $(\alpha, \beta)$ have identical $S_{11}$, i.e.,

$$
S_{11}\left(\alpha, \beta, S_{i j}^{(0)}\right)=S_{11}^{(0)}
$$

From the relation between the original compliance matrix and the rotated one (see Appendix A), the detailed expression of Eq. (3.9) can be obtained as

$$
\begin{aligned}
& 0=S_{11}\left(\alpha, \beta, S_{i j}^{(0)}\right)-S_{11}^{(0)} \\
& =\left(\cos ^{4}(\beta)-1\right) S_{11}^{(0)}+\left[1-\cos ^{2}(\alpha)\right]^{2}\left[1-\cos ^{2}(\beta)\right]^{2} S_{22}^{(0)}+\cos ^{4}(\alpha)\left[1-\cos ^{2}(\beta)\right]^{2} S_{33}^{(0)} \\
& +\sin ^{2}(\alpha) \cos ^{2}(\alpha)\left[1-\cos ^{2}(\beta)\right]^{2}\left(S_{44}^{(0)}+2 S_{23}^{(0)}\right) \\
& +\cos ^{2}(\alpha) \sin ^{2}(\beta) \cos ^{2}(\beta)\left(S_{55}^{(0)}+2 S_{13}^{(0)}\right) \\
& +\sin ^{2}(\alpha) \sin ^{2}(\beta) \cos ^{2}(\beta)\left(S_{66}^{(0)}+2 S_{12}^{(0)}\right) \\
& -2 \sin (\alpha) \cos (\alpha) \sin ^{2}(\beta) \cos ^{2}(\beta)\left(S_{14}^{(0)}+S_{56}^{(0)}\right) \\
& -2 \sin ^{2}(\alpha) \cos (\alpha) \sin ^{3}(\beta) \cos (\beta)\left(S_{25}^{(0)}+S_{46}^{(0)}\right) \\
& +2 \sin (\alpha) \cos ^{2}(\alpha) \sin ^{3}(\beta) \cos (\beta)\left(S_{36}^{(0)}+S_{45}^{(0)}\right) \\
& -2 \cos (\alpha) \sin (\beta) \cos ^{3}(\beta) S_{15}^{(0)}+2 \sin (\alpha) \sin (\beta) \cos ^{3}(\beta) S_{16}^{(0)} \\
& -2 \sin ^{3}(\alpha) \cos (\alpha)\left[1-\cos ^{2}(\beta)\right]^{2} S_{24}^{(0)} \\
& +2 \sin ^{3}(\alpha) \sin ^{3}(\beta) \cos (\beta) S_{26}^{(0)}-2 \sin (\alpha) \cos ^{3}(\alpha)\left[1-\cos ^{2}(\beta)\right]^{2} S_{34}^{(0)} \\
& -2 \cos ^{3}(\alpha) \sin ^{3}(\beta) \cos (\beta) S_{35}^{(0)} .
\end{aligned}
$$

For any $\alpha$ and $\beta$, the following relations can satisfy Eq. (3.10),

$$
\begin{aligned}
& S_{11}^{(0)}=S_{22}^{(0)}=S_{33}^{(0)}, \quad S_{15}^{(0)}=S_{16}^{(0)}=S_{24}^{(0)}=S_{26}^{(0)}=S_{34}^{(0)}=S_{35}^{(0)}=0, \\
& S_{14}^{(0)}+S_{56}^{(0)}=S_{25}^{(0)}+S_{46}^{(0)}=S_{36}^{(0)}+S_{45}^{(0)}=0, \\
& S_{44}^{(0)}=2\left(S_{11}^{(0)}-S_{23}^{(0)}\right), \quad S_{55}^{(0)}=2\left(S_{11}^{(0)}-S_{13}^{(0)}\right), \quad S_{66}^{(0)}=2\left(S_{11}^{(0)}-S_{12}^{(0)}\right),
\end{aligned}
$$


or expressed in the compliance matrix form

$$
\begin{aligned}
& \mathbf{S}_{X_{1}-X_{2}-X_{3}} \\
& \quad=\left[\begin{array}{cccccc}
S_{11}^{(0)} & S_{12}^{(0)} & S_{13}^{(0)} & S_{14}^{(0)} & 0 & 0 \\
& S_{11}^{(0)} & S_{23}^{(0)} & 0 & S_{25}^{(0)} & 0 \\
& & S_{11}^{(0)} & 0 & 0 & S_{36}^{(0)} \\
& (\mathrm{sym}) & & 2\left(S_{11}^{(0)}-S_{23}^{(0)}\right) & -S_{36}^{(0)} & -S_{25}^{(0)} \\
& & & & 2\left(S_{11}^{(0)}-S_{13}^{(0)}\right) & -S_{14}^{(0)} \\
& & & & & 2\left(S_{11}^{(0)}-S_{12}^{(0)}\right)
\end{array}\right] .
\end{aligned}
$$

Obviously, Eq. (3.12) is not consistent with the following compliance matrix for the isotropic symmetry of material elastic properties.

$$
\mathbf{S}^{\text {isotropic }}=\left[\begin{array}{cccccc}
S_{11}^{(0)} & S_{12}^{(0)} & S_{12}^{(0)} & 0 & 0 & 0 \\
& S_{11}^{(0)} & S_{12}^{(0)} & 0 & 0 & 0 \\
& & S_{11}^{(0)} & 0 & 0 & 0 \\
& & & 2\left(S_{11}^{(0)}-S_{12}^{(0)}\right) & 0 & 0 \\
& & & & 2\left(S_{11}^{(0)}-S_{12}^{(0)}\right) & 0 \\
& & & & 2\left(S_{11}^{(0)}-S_{12}^{(0)}\right)
\end{array}\right] .
$$

It is very interesting to note from Eqs. (3.12) and (3.13) that a material with isotropic tensile stiffness is not definitely an isotropic elastic material. In another word, the symmetry of the material elastic properties cannot be completely identified by only measuring the tensile stiffness of all directions. More interestingly, these statements are only correct for three-dimensional elastic constitutive relations, but do not hold for two-dimensional ones. Namely, an isotropic symmetry of tensile stiffness in two dimensions is definitely equivalent to an isotropic symmetry of material elastic properties. The detailed analytic proof on two-dimensional cases is presented in Appendix C.

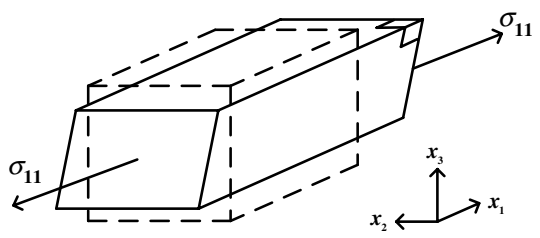

Fig. 9. The deformation of a cuboid RVE subject to a uniaxial tensile stress $\sigma_{11}$. 
For three-dimensional materials with an isotropic symmetry of tensile stiffness, Fig. 9 shows a schematic example, a cuboid RVE subject to a uniaxial tensile stress $\sigma_{11}$. From the interpretation context related to Fig. 3, we know $S_{15}=S_{16}=0$, since every direction is the stiffest direction when the RVE possesses isotropic symmetry of tensile stiffness. However, a nonvanishing shear deformation $\gamma_{23}$ normal to the tensile stress $\sigma_{11}$ cannot be excluded by those conditions, resulting in a nonvanishing compliance component $S_{14}$ and anisotropy of the material.

\section{A Simple and Correct Measure of Anisotropy Degree}

The last part of the previous section exhibits a possibility that, at least in mathematical sense, a material with isotropic tensile stiffness does not have isotropic elastic properties. In some studies based on representative volume element [e.g., Segurado and Llorca, 2002; Guo et al., 2014], the isotropy of elastic properties is required, and a proper measure is needed to evaluate it quickly. However, many researchers have incorrectly adopted the ratio between the maximum and minimum tensile stiffnesses as the measure of anisotropy degree [Ni and Chiang, 2007; Margenov et al., 2014]. In the following, we will construct a periodical lattice structure to demonstrate the failure of this measure in identifying the anisotropy.

\subsection{An anisotropic material with isotropic tensile stiffness}

Figure 10 shows a periodic cubic unit cell (or a representative volume element) of a lattice structure with side length $l$, which consists of two-force bars and connecting nodes. One node is inside the cube and eight other nodes are located at the corners. An optimization is carried out on the stiffnesses of bars and the position of the inside node to make its tensile stiffness isotropic while keeping the anisotropy of material elasticity, and the results are given in Table 1 . The corresponding effective compliance matrix $\mathbf{S}$ of this latticed material is given in $X_{1}-X_{2}-X_{3}$ coordinate

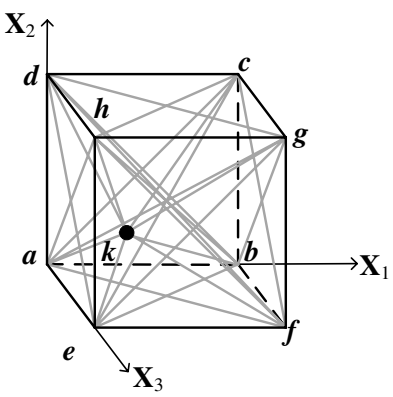

Fig. 10. A periodic cubic unit cell of a lattice structure with two-force bars and connecting nodes. 
Table 1. The optimization results of the stiffnesses of bars and the position of the inside node.

\begin{tabular}{lrrr}
\hline The position of the inside node & $\left(\frac{x_{1}^{k}}{l}, \frac{x_{2}^{k}}{l}, \frac{x_{3}^{k}}{l}\right)=(0.7866,0.6736,0.2795)$ \\
\hline The bar stiffness & $k^{a b}=k^{a b}$ & $k^{a c}=0.3727 k^{a b}$ & $k^{a k}=0.6465 k^{a b}$ \\
& $k^{b c}=0.9566 k^{a b}$ & $k^{b d}=0.2918 k^{a b}$ & $k^{b k}=1.8602 k^{a b}$ \\
$k^{a e}=0.9781 k^{a b}$ & $k^{c h}=0.3782 k^{a b}$ & $k^{c k}=1.1756 k^{a b}$ \\
$k^{a g}=0.8179 k^{a b}$ & $k^{d g}=0.3114 k^{a b}$ & $k^{d k}=1.6885 k^{a b}$ \\
& $k^{b h}=0.8451 k^{a b}$ & $k^{a h}=0.2173 k^{a b}$ & $k^{e k}=1.6332 k^{a b}$ \\
$k^{c e}=0.4116 k^{a b}$ & $k^{d e}=0.3331 k^{a b}$ & $k^{f k}=1.0263 k^{a b}$ \\
& $k^{d f}=0.7512 k^{a b}$ & & $k^{g k}=2.0571 k^{a b}$ \\
& & & $k^{h k}=0.5734 k^{a b}$ \\
\hline
\end{tabular}

system as

$$
\mathbf{S}=\frac{l}{k^{a b}}\left[\begin{array}{cccccc}
0.1763 & -0.0434 & -0.0426 & -0.0111 & 0 & 0 \\
& 0.1763 & -0.0436 & 0 & -0.0066 & 0 \\
& & 0.1763 & 0 & 0 & 0.0080 \\
& (\mathrm{sym}) & & 0.4399 & -0.0080 & 0.0066 \\
& & & & 0.4378 & 0.0111 \\
& & & & & 0.4395
\end{array}\right]
$$

where $k^{a b}$ is the stiffness of the bar connecting node $a$ and node $b$.

Obviously, the tensile stiffnesses along $X_{1^{-}}, X_{2^{-}}$and $X_{3}$-directions are the same, but this compliance matrix is not an isotropic one. Furthermore, the finite element software ABAQUS is adopted to test the isotropy of the tensile stiffness along any direction. Four arbitrary directions denoted by Euler angles $(\alpha, \beta, \gamma$ as shown in Fig. 8) are chosen to simulate the uniaxial tensile tests, and the stresses and strains both in the reference $X_{1}-X_{2}-X_{3}$ coordinate system and corresponding rotated coordinate systems are given in Table 2 . It is easy to apply loading in the reference $X_{1}-X_{2}-X_{3}$ coordinate system and to obtain the tensile stiffness in the rotated coordinate system. The first component of the strain matrix in the rotated coordinate system clearly exhibits the isotropy of the tensile stiffness. Hence, this is a counter example that demonstrates the invalidity of the measure of anisotropy degree based on the ratio between the maximum and minimum tensile stiffnesses. A more proper measure is needed.

\subsection{A measure of anisotropy degree based on the maximum shear-extension coupling coefficient}

As mentioned in Sec. 3, a nonvanishing shear deformation $\gamma_{23}$ cannot be excluded in a material with only isotropic tensile stiffness. In this paper, we therefore propose the maximum ratio between the shear and tensile strain under uniaxial tension in all orientations, i.e., the maximum shear-extension coupling coefficient, as a simple 


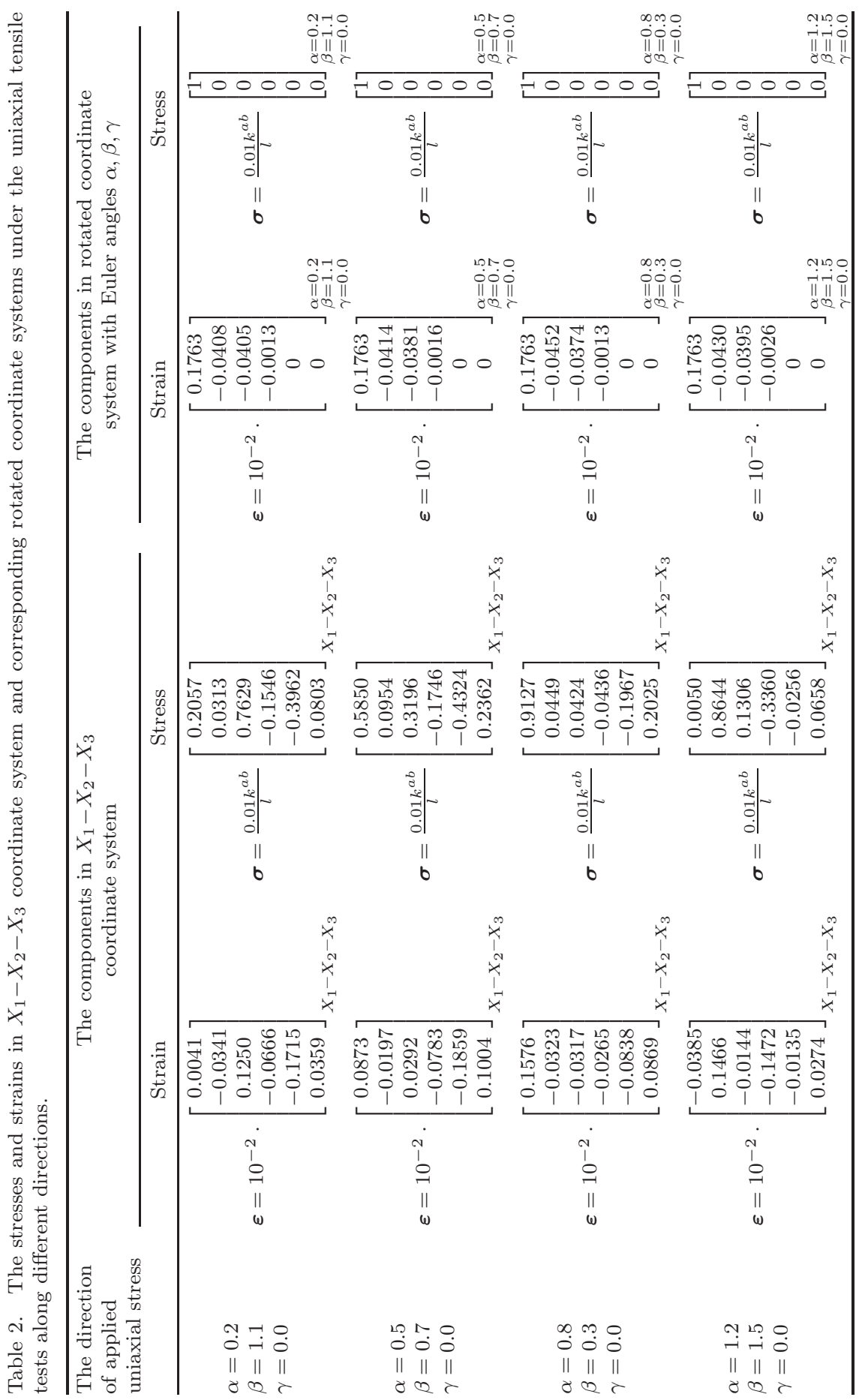


measure of anisotropy degree,

$$
\eta_{\text {max }}=\max \left(\frac{\gamma_{\text {normal }}}{\varepsilon_{\text {tensile }}}, \frac{\gamma_{\text {in-plane }}}{\varepsilon_{\text {tensile }}}\right)_{\text {for all orientations }},
$$

where $\gamma_{\text {normal }}=\gamma_{23}$ and $\gamma_{\text {in-plane }}=\gamma_{12}$ are shear strains normal to and in the plane of the uniaxial tensile direction ( $x_{1}$-direction), respectively, and $\varepsilon_{\text {tensile }}=\varepsilon_{11}$ is the tensile strain. $\eta_{\max }$ can also be expressed with components of compliance matrix as

$$
\eta_{\max }=\max \left(\frac{S_{14}}{S_{11}}, \frac{S_{16}}{S_{11}}\right)_{\text {for all coordinate systems }} .
$$

When a material subject to uniaxial stretcthing in any direction has no shear deformation, $\eta_{\max }$ is zero, and we will prove in the following that the material has isotropic elaticity.

According to the relevant analysis of Fig. 3 and Eqs. (2.6) and (2.7), no in-plane shear strain for uniaxial stretcthing in any direction implies that the derivative of the tensile stiffness along any direction with respect to the orientation angle is zero, and the material has isotropic tensile stiffness. Therefore, the corresponding compliance matrix has the form as Eq. (3.12). Still based on zero shear strain,

$$
S_{14}^{(0)}=S_{25}^{(0)}=S_{36}^{(0)}=0 .
$$

The compliance matrix becomes

$$
\begin{aligned}
& \mathbf{S}_{X_{1}-X_{2}-X_{3}} \\
& \quad=\left[\begin{array}{ccccccc}
S_{11}^{(0)} & S_{12}^{(0)} & S_{13}^{(0)} & 0 & 0 & 0 \\
& S_{11}^{(0)} & S_{23}^{(0)} & 0 & 0 & 0 \\
& & S_{11}^{(0)} & 0 & 0 & 0 \\
& & & 2\left(S_{11}^{(0)}-S_{23}^{(0)}\right) & 0 & 0 \\
& & & 2\left(S_{11}^{(0)}-S_{13}^{(0)}\right) & 0 \\
& & & & 2\left(S_{11}^{(0)}-S_{12}^{(0)}\right)
\end{array}\right] .
\end{aligned}
$$

If $S_{12}^{(0)} \neq S_{13}^{(0)}, \varepsilon_{22} \neq \varepsilon_{33}$ for the material under $X_{1}$-direction tension, and nonzero shear strain will appear when the coordinate system rotates around $X_{1}$-axis. Therefore, $S_{12}^{(0)}=S_{13}^{(0)}$. Similarly, $S_{12}^{(0)}=S_{23}^{(0)}$. Equation (4.5) is then the same as the isotropic elastic compliance matrix equation (3.13). It is proved that this maximum shear-extension coupling coefficient $\eta_{\max }$ is a correct and simple measure of anisotropy degree.

It must be pointed out that all the conclusions in this paper are achieved through simple matrix operations, without using other advanced knowledge such as the symmetry of point group and tensor analysis, which is therefore easy to understand. These related issues have also been investigated via tensor analysis. For example, 
He [2004], Ting [2006] and Ting and He [2006] discussed the general forms of elastic tensors with orientation-independent tensile stiffness through decomposition of elasticity tensors. Moreover, He [2004] pointed out that the elastic properties is isotropic if only the Young's modulus and traction-associated bulk modulus are both isotropic, which may serve as another measure of anisotropy degree. There are also some measures based on the norm of elastic tensor [Norris, 2006; Alhayek and Gaith, 2010]. It is easy to find that among all these measures, the proposed measure of anisotropy degree based on the maximum shear-extension coupling coefficient is simpler and has more straightforward physical meanings.

\section{Conclusion}

In this paper, standardized compliance matrices for general anisotropic materials and a new measure of anisotropy degree have been proposed and discussed. The following conclusions are achieved.

(1) For a general anisotropic material, a unique stiffest orientation-based standardized compliance matrix is proposed, which clearly exhibits 18 independent elastic material constants and makes it convenient to compare the elastic properties between different materials.

(2) If a material has some orientational symmetry, the symmetry orientation-based standardized compliance matrix can also be obtained, which can show the reduced independent elastic material constants. During the searching process for the symmetry, it is interesting to find that a material with some symmetry of the tensile stiffness, such as isotropic symmetry, does not definitely possess the same symmetry in its elastic properties.

(3) It is important to find that the ratio between the maximum and minimum tensile stiffnesses is not a correct measure of anisotropy degree. The maximum shear-extension coupling coefficient in all orientations is proposed to serve as a simple and correct measure of anisotropy degree. Therefore, the most essential feature of isotropic elasticity is no shear-extension coupling, not isotropic tensile stiffness. However, for a two-dimensional constitutive relation, both the stiffness ratio and the shear-extension coupling coefficient can be adopted as proper measures of anisotropy degree.

\section{Acknowledgment}

The authors acknowledge the support from National Natural Science Foundation of China (Grant Nos. 11425208, 51232004 and 11372158) and Tsinghua University Initiative Scientific Research Program (No. 2011Z02173).The authors are grateful for helpful discussions with Professor QuanShui Zheng and Professor Qichang He. 


\section{Appendix A}

Assume the reference coordinate system is denoted as $X_{1}-X_{2}-X_{3}$, and the rotated coordinate system denoted as $x_{1}^{(1)}-x_{2}^{(1)}-x_{3}^{(1)}$ is obtained by rotating the reference coordinate system angle $\theta_{1}$ around $X_{1}$-axis. The corresponding stress and strain matrices in these two coordinate systems have the following relation,

$$
\boldsymbol{\sigma}^{(1)}=\mathbf{T}_{\theta_{1}}^{\sigma} \cdot \boldsymbol{\sigma}^{(0)}, \quad \boldsymbol{\varepsilon}^{(1)}=\mathbf{T}_{\theta_{1}}^{\varepsilon} \cdot \boldsymbol{\varepsilon}^{(0)},
$$

where $\mathbf{T}_{\theta_{1}}^{\sigma}$ and $\mathbf{T}_{\theta_{1}}^{\varepsilon}$ are the stress and strain transformation matrices, respectively, and can be written as

$$
\begin{aligned}
& \mathbf{T}_{\theta_{1}}^{\sigma}=\left[\begin{array}{cccccc}
1 & 0 & 0 & 0 & 0 & 0 \\
0 & \cos ^{2}\left(\theta_{1}\right) & \sin ^{2}\left(\theta_{1}\right) & 2 \sin \left(\theta_{1}\right) \cos \left(\theta_{1}\right) & 0 & 0 \\
0 & \sin ^{2}\left(\theta_{1}\right) & \cos ^{2}\left(\theta_{1}\right) & -2 \sin \left(\theta_{1}\right) \cos \left(\theta_{1}\right) & 0 & 0 \\
0 & -\sin \left(\theta_{1}\right) \cos \left(\theta_{1}\right) & \sin \left(\theta_{1}\right) \cos \left(\theta_{1}\right) & \cos ^{2}\left(\theta_{1}\right)-\sin ^{2}\left(\theta_{1}\right) & 0 & 0 \\
0 & 0 & 0 & 0 & \cos \left(\theta_{1}\right) & -\sin \left(\theta_{1}\right) \\
0 & 0 & 0 & 0 & \sin \left(\theta_{1}\right) & \cos \left(\theta_{1}\right)
\end{array}\right], \\
& \mathbf{T}_{\theta_{1}}^{\varepsilon}=\left[\begin{array}{cccccc}
1 & 0 & 0 & 0 & 0 & 0 \\
0 & \cos ^{2}\left(\theta_{1}\right) & \sin ^{2}\left(\theta_{1}\right) & \sin \left(\theta_{1}\right) \cos \left(\theta_{1}\right) & 0 & 0 \\
0 & \sin ^{2}\left(\theta_{1}\right) & \cos ^{2}\left(\theta_{1}\right) & -\sin \left(\theta_{1}\right) \cos \left(\theta_{1}\right) & 0 & 0 \\
0 & -2 \sin \left(\theta_{1}\right) \cos \left(\theta_{1}\right) & 2 \sin \left(\theta_{1}\right) \cos \left(\theta_{1}\right) & \cos ^{2}\left(\theta_{1}\right)-\sin ^{2}\left(\theta_{1}\right) & 0 & 0 \\
0 & 0 & 0 & 0 & \cos \left(\theta_{1}\right) & -\sin \left(\theta_{1}\right) \\
0 & 0 & 0 & 0 & \sin \left(\theta_{1}\right) & \cos \left(\theta_{1}\right)
\end{array}\right] .
\end{aligned}
$$

Similarly, when rotating the coordinate system $X_{1}-X_{2}-X_{3}$ around $X_{2}$-axis by an angle $\theta_{2}$, the stress and strain transformation matrices in the rotated coordinate system $x_{1}^{(2)}-x_{2}^{(2)}-x_{3}^{(2)}, \mathbf{T}_{\theta_{2}}^{\sigma}$ and $\mathbf{T}_{\theta_{2}}^{\varepsilon}$, can be written

$$
\mathbf{T}_{\theta_{2}}^{\boldsymbol{\sigma}}=\left[\begin{array}{cccccc}
\cos ^{2}\left(\theta_{2}\right) & 0 & \sin ^{2}\left(\theta_{2}\right) & 0 & -2 \sin \left(\theta_{2}\right) \cos \left(\theta_{2}\right) & 0 \\
0 & 1 & 0 & 0 & 0 & 0 \\
\sin ^{2}\left(\theta_{2}\right) & 0 & \cos ^{2}\left(\theta_{2}\right) & 0 & 2 \sin \left(\theta_{2}\right) \cos \left(\theta_{2}\right) & 0 \\
0 & 0 & 0 & \cos \left(\theta_{2}\right) & 0 & \sin \left(\theta_{2}\right) \\
\sin \left(\theta_{2}\right) \cos \left(\theta_{2}\right) & 0 & -\sin \left(\theta_{2}\right) \cos \left(\theta_{2}\right) & 0 & \cos ^{2}\left(\theta_{2}\right)-\sin ^{2}\left(\theta_{2}\right) & 0 \\
0 & 0 & 0 & -\sin \left(\theta_{2}\right) & 0 & \cos \left(\theta_{2}\right)
\end{array}\right],
$$




$$
\mathbf{T}_{\theta_{2}}^{\varepsilon}=\left[\begin{array}{cccccc}
\cos ^{2}\left(\theta_{2}\right) & 0 & \sin ^{2}\left(\theta_{2}\right) & 0 & -\sin \left(\theta_{2}\right) \cos \left(\theta_{2}\right) & 0 \\
0 & 1 & 0 & 0 & 0 & 0 \\
\sin ^{2}\left(\theta_{2}\right) & 0 & \cos ^{2}\left(\theta_{2}\right) & 0 & \sin \left(\theta_{2}\right) \cos \left(\theta_{2}\right) & 0 \\
0 & 0 & 0 & \cos \left(\theta_{2}\right) & 0 & \sin \left(\theta_{2}\right) \\
2 \sin \left(\theta_{2}\right) \cos \left(\theta_{2}\right) & 0 & -2 \sin \left(\theta_{2}\right) \cos \left(\theta_{2}\right) & 0 & \cos ^{2}\left(\theta_{2}\right)-\sin ^{2}\left(\theta_{2}\right) & 0 \\
0 & 0 & 0 & -\sin \left(\theta_{2}\right) & 0 & \cos \left(\theta_{2}\right)
\end{array}\right] .
$$

When rotating the coordinate system $X_{1}-X_{2}-X_{3}$ around $X_{3}$-axis by an angle $\theta_{3}$, the stress and strain transformation matrices in the rotated coordinate system $x_{1}^{(3)}-x_{2}^{(3)}-x_{3}^{(3)}, \mathbf{T}_{\theta_{3}}^{\boldsymbol{\sigma}}$ and $\mathbf{T}_{\theta_{3}}^{\boldsymbol{\varepsilon}}$, can be written as

$$
\begin{array}{r}
\mathbf{T}_{\theta_{3}}^{\boldsymbol{\sigma}}=\left[\begin{array}{cccccc}
\cos ^{2}\left(\theta_{3}\right) & \sin ^{2}\left(\theta_{3}\right) & 0 & 0 & 0 & 2 \sin \left(\theta_{3}\right) \cos \left(\theta_{3}\right) \\
\sin ^{2}\left(\theta_{3}\right) & \cos ^{2}\left(\theta_{3}\right) & 0 & 0 & 0 & -2 \sin \left(\theta_{3}\right) \cos \left(\theta_{3}\right) \\
0 & 0 & 1 & 0 & 0 & 0 \\
0 & 0 & 0 & \cos \left(\theta_{3}\right) & -\sin \left(\theta_{3}\right) & 0 \\
0 & 0 & 0 & \sin \left(\theta_{3}\right) & \cos \left(\theta_{3}\right) & 0 \\
-\sin \left(\theta_{3}\right) \cos \left(\theta_{3}\right) & \sin \left(\theta_{3}\right) \cos \left(\theta_{3}\right) & 0 & 0 & 0 & \cos ^{2}\left(\theta_{3}\right)-\sin ^{2}\left(\theta_{3}\right)
\end{array}\right], \\
\mathbf{T}_{\theta_{3}}^{\boldsymbol{\varepsilon}}=\left[\begin{array}{cccccc}
\cos ^{2}\left(\theta_{3}\right) & \sin ^{2}\left(\theta_{3}\right) & 0 & 0 & 0 & \sin \left(\theta_{3}\right) \cos \left(\theta_{3}\right) \\
\sin ^{2}\left(\theta_{3}\right) & \cos ^{2}\left(\theta_{3}\right) & 0 & 0 & 0 & -\sin \left(\theta_{3}\right) \cos \left(\theta_{3}\right) \\
0 & 0 & 1 & 0 & 0 & 0 \\
0 & 0 & 0 & \cos \left(\theta_{3}\right) & -\sin \left(\theta_{3}\right) & 0 \\
0 & 0 & 0 & \sin \left(\theta_{3}\right) & \cos \left(\theta_{3}\right) & 0 \\
-2 \sin \left(\theta_{3}\right) \cos \left(\theta_{3}\right) & 2 \sin \left(\theta_{3}\right) \cos \left(\theta_{3}\right) & 0 & 0 & 0 & \cos ^{2}\left(\theta_{3}\right)-\sin ^{2}\left(\theta_{3}\right)
\end{array}\right] .
\end{array}
$$

According to Eq. (1.1), the relation between compliance matrices $\mathbf{S}^{(0)}$ in $X_{1}-X_{2}-X_{3}$ and $\mathbf{S}^{(i)}$ in $x_{1}^{(i)}-x_{2}^{(i)}-x_{3}^{(i)}$ can be obtained as

$$
\mathbf{S}^{(i)}=\mathbf{T}_{\theta_{i}}^{\varepsilon} \cdot \mathbf{S}^{(0)} \cdot\left(\mathbf{T}_{\theta_{i}}^{\sigma}\right)^{-1} .
$$

In Sec. 2.1, Coordinate system (b), (c) and (d) can be obtained through rotating Coordinate system (a) $\pi$ angle around $\hat{x}_{1}^{a}, \hat{x}_{2}^{a}$ - and $\hat{x}_{3}^{a}$-axis, respectively. Therefore, the compliance matrices $\mathbf{S}^{b}, \mathbf{S}^{c}$ and $\mathbf{S}^{d}$ can be expressed as

$$
\begin{aligned}
& \mathbf{S}^{b}=\mathbf{T}_{\theta_{1}=\pi}^{\varepsilon} \cdot \mathbf{S}^{a} \cdot\left(\mathbf{T}_{\theta_{1}=\pi}^{\sigma}\right)^{-1}, \\
& \mathbf{S}^{c}=\mathbf{T}_{\theta_{2}=\pi}^{\varepsilon} \cdot \mathbf{S}^{a} \cdot\left(\mathbf{T}_{\theta_{2}=\pi}^{\sigma}\right)^{-1}, \\
& \mathbf{S}^{d}=\mathbf{T}_{\theta_{3}=\pi}^{\varepsilon} \cdot \mathbf{S}^{a} \cdot\left(\mathbf{T}_{\theta_{3}=\pi}^{\sigma}\right)^{-1} .
\end{aligned}
$$


In Sec. 3.2, $S_{11}\left(\alpha, \beta, S_{i j}^{(0)}\right)$ is the tensile compliance component along the direction expressed by Euler angles $(\alpha, \beta)$ and the corresponding compliance matrix is

$$
\mathbf{S}\left(\alpha, \beta, S_{i j}^{(0)}\right)=\left(\mathbf{T}_{\theta_{2}=\beta}^{\varepsilon}\right) \cdot\left(\mathbf{T}_{\theta_{1}=\alpha}^{\varepsilon}\right) \cdot \mathbf{S}^{(0)} \cdot\left(\mathbf{T}_{\theta_{1}=\alpha}^{\sigma}\right)^{-1} \cdot\left(\mathbf{T}_{\theta_{2}=\beta}^{\sigma}\right)^{-1} .
$$

\section{Appendix B}

From Fig. 6, the process of rotating the coordinate system $\hat{x}_{1}^{(1)}-\hat{x}_{2}^{(1)}-\hat{x}_{3}^{(1)}$ to $\hat{x}_{1}^{(2)}-\hat{x}_{2}^{(2)}-\hat{x}_{3}^{(2)}$ is as follows: firstly, rotating coordinate system $\hat{x}_{1}^{(1)}-\hat{x}_{2}^{(1)}-\hat{x}_{3}^{(1)}$ around $\hat{x}_{1}^{(1)}$-axis by an angle $-\Theta^{(1)}$; then, rotating coordinate system $\hat{x}_{1}^{(1)}-x_{2}^{(1)}-x_{3}^{(1)}$ around $x_{3}^{(1)}$-axis by an angle $-2 \varphi$; finally, rotating coordinate system $\hat{x}_{1}^{(2)}-x_{2}^{(1)^{\prime}}-x_{3}^{(1)}$ around $\hat{x}_{1}^{(2)}$-axis by an angle $\pi+\Theta^{(2)}$. Considering that the compliance matrix $\hat{\mathbf{S}}$ under the two STF standardized coordinate systems are identical, according to transformation relation of compliance matrices under different coordinate systems Eq. (A.5), we have

$$
\begin{aligned}
\hat{\mathbf{S}}= & \left(\mathbf{T}_{\theta_{1}=\pi+\Theta^{(2)}}^{\varepsilon}\right) \cdot\left(\mathbf{T}_{\theta_{3}=-2 \varphi}^{\varepsilon}\right) \cdot\left(\mathbf{T}_{\theta_{1}=-\Theta^{(1)}}^{\varepsilon}\right) \cdot \hat{\mathbf{S}} \cdot\left(\mathbf{T}_{\theta_{1}=-\Theta^{(1)}}^{\sigma}\right)^{-1} \\
& \cdot\left(\mathbf{T}_{\theta_{3}=-2 \varphi}^{\sigma}\right)^{-1} \cdot\left(\mathbf{T}_{\theta_{1}=\pi+\Theta^{(2)}}^{\sigma}\right)^{-1} .
\end{aligned}
$$

Similarly, rotating the coordinate system $\hat{x}_{1}^{(2)}-\hat{x}_{2}^{(2)}-\hat{x}_{3}^{(2)}$ to $\hat{x}_{1}^{(1)}-\hat{x}_{2}^{(1)}-\hat{x}_{3}^{(1)}$ leads to the following relation

$$
\begin{aligned}
\hat{\mathbf{S}}= & \left(\mathbf{T}_{\theta_{1}=\pi+\Theta^{(1)}}^{\varepsilon}\right) \cdot\left(\mathbf{T}_{\theta_{3}=-2 \varphi}^{\varepsilon}\right) \cdot\left(\mathbf{T}_{\theta_{1}=-\Theta^{(2)}}^{\varepsilon}\right) \cdot \hat{\mathbf{S}} \cdot\left(\mathbf{T}_{\theta_{1}=-\Theta^{(2)}}^{\sigma}\right)^{-1} \\
& \cdot\left(\mathbf{T}_{\theta_{3}=-2 \varphi}^{\sigma}\right)^{-1} \cdot\left(\mathbf{T}_{\theta_{1}=\pi+\Theta^{(1)}}^{\sigma}\right)^{-1} .
\end{aligned}
$$

From Eqs. (B.1) and (B.2), it can be inferred that

$$
\left(\mathbf{T}_{\theta_{1}=\pi+\Theta^{(2)}}^{\varepsilon}\right) \cdot\left(\mathbf{T}_{\theta_{3}=-2 \varphi}^{\varepsilon}\right) \cdot\left(\mathbf{T}_{\theta_{1}=-\Theta^{(1)}}^{\varepsilon}\right)=\left(\mathbf{T}_{\theta_{1}=\pi+\Theta^{(1)}}^{\varepsilon}\right) \cdot\left(\mathbf{T}_{\theta_{3}=-2 \varphi}^{\varepsilon}\right) \cdot\left(\mathbf{T}_{\theta_{1}=-\Theta^{(2)}}^{\varepsilon}\right)
$$

and

$$
\Theta_{1}=\Theta_{2} \text {. }
$$

\section{Appendix C}

Assume that a two-dimensional material is in the plane $X_{1}-X_{2}$ and the orientational variation of $S_{11}$ in the plane $X_{1}-X_{2}$ is isotropic. When rotating the coordinate system $X_{1}-X_{2}$ by any angle $\theta_{3}$, according to Eq. (A.5), the rotated compliance matrix $\mathbf{S}_{2 D}\left(\theta_{3}, S_{i j}^{(0)}\right)$ can be written as

$$
\mathbf{S}_{2 D}\left(\theta_{3}, S_{i j}^{(0)}\right)=\mathbf{T}_{\theta_{3}}^{\varepsilon} \cdot \mathbf{S}_{2 D}^{(0)} \cdot\left(\mathbf{T}_{\theta_{3}}^{\boldsymbol{\sigma}}\right)^{-1}
$$

and the value of component $S_{11}$ is always the same, i.e.,

$$
S_{11}\left(\theta_{3}, S_{i j}^{(0)}\right)=S_{11}^{(0)} .
$$


From Eq. (A.5), the above equation can be expressed in detail as

$$
\begin{aligned}
0= & S_{11}\left(\theta_{3}, S_{i j}^{(0)}\right)-S_{11}^{(0)} \\
= & {\left[\cos ^{4}\left(\theta_{3}\right)-1\right] S_{11}^{(0)}+\sin ^{4}\left(\theta_{3}\right) S_{22}^{(0)}+2 \sin \left(\theta_{3}\right) \cos ^{3}\left(\theta_{3}\right) S_{16}^{(0)} } \\
& +2 \sin ^{3}\left(\theta_{3}\right) \cos \left(\theta_{3}\right) S_{26}^{(0)}+\sin ^{2}\left(\theta_{3}\right) \cos ^{2}\left(\theta_{3}\right)\left(S_{66}^{(0)}+2 S_{12}^{(0)}\right) .
\end{aligned}
$$

When $\theta_{3}=\pi / 2$, it can be easily inferred that $S_{11}^{(0)}=S_{22}^{(0)}$. Thus, Eq. (C.3) can be simplified as

$$
0=\sin \left(\theta_{3}\right) \cos \left(\theta_{3}\right)\left(S_{66}^{(0)}+2 S_{12}^{(0)}-2 S_{11}^{(0)}\right)+2 \cos ^{2}\left(\theta_{3}\right) S_{16}^{(0)}+2 \sin ^{2}\left(\theta_{3}\right) S_{26}^{(0)} .
$$

Then, the following relations can be inferred

$$
S_{11}^{(0)}=S_{22}^{(0)}, \quad S_{66}^{(0)}=2\left(S_{11}^{(0)}-S_{12}^{(0)}\right), \quad S_{16}^{(0)}=S_{26}^{(0)}=0 .
$$

This implies that a two-dimensional material with the isotropic symmetry of the tensile stiffness definitely possess the same isotropic symmetry in its elastic properties.

\section{References}

Alhayek, I. and Gaith, M. S. [2010] "The measurement of overall elastic stiffness and bulk modulus in solar photovoltaic devices," Journal of Mechanics of Materials and Structures 4(1), 55-60.

Boulanger, P. and Hayes, M. [1995] "On Young's modulus for anisotropic media," Journal of Applied Mechanics 62(3), 819-820.

Bower, A. F. [2011] Applied Mechanics of Solids (CRC Press, New York).

Cazzani, A. and Rovati, M. [2003] "Extrema of Young's modulus for cubic and transversely isotropic solids," International Journal of Solids and Structures 40(7), 1713-1744.

Cowin, S. C. and Mehrabadi, M. M. [1992] "The structure of the linear anisotropic elastic symmetries," Journal of Mechanics and Physics of Solids 40(7), 1459-1471.

Cowin, S. C. and Mehrabadi, M. M. [1995] "Anisotropic symmetries of linear elasticity," Applied Mechanics Reviews 48(5), 247-285.

Cowin, S. C. [1995] "On the number of distinct elastic constants associated with certain anisotropic elastic symmetries," Zeitschrift fur Angewandte Mathematik und Physik 46, S210-S224.

Dellinger, J., Vasicek, D. and Sondergeld C. [1998] "Kelvin notation for stabilizing elasticconstant inversion," Oil \& Gas Science and Technology 53(5), 709-719.

Fedorov, F. I. [1968] Theory of Elastic Waves in Crystals (Plenum Press, New York).

Guo, Z., Shi, X., Chen, Y., Chen, H., Peng, X. and Harrison, P. [2014] "Mechanical modeling of incompressible particle-reinforced neo-Hookean composites based on numerical homogenization," Mechanics of Materials 70, 1-17.

He, Q. C. [2004] "Characterization of the anisotropic materials capable of exhibiting an isotropic Young or shear or area modulus," International Journal of Engineering Science 42(19), 2107-2118.

Herakovich, C. T. [2012] "Mechanics of composites: A historical review," Mechanics Research Communications 41, 1-20.

Jones, R. M. [1999] Mechanics of Composite Materials, 2nd edn. (Taylor \& Francis, Philadelphia). 
Kaw, A. K. [1997] Mechanics of Composite Materials (CRC Press, New York).

Kowalczyk-Gajewska, K. and Ostrowska-Maciejewska, J. [2009] "Review on spectral decomposition of Hooke's tensor for all symmetry groups of linear elastic material," Engineering Transactions 57(3-4), 145-183.

Margenov, S., Stoykov, S. and Vutov, Y. [2014] "Numerical homogenization of heterogeneous anisotropic linear elastic materials," Large-Scale Scientific Computing: 9th International Conference, Bulgaria, pp. 347-354.

Ni, Y. and Chiang, M. Y. [2007] "Prediction of elastic properties of heterogeneous materials with complex microstructures," Journal of Mechanics and Physics of Solids 55(3), $517-532$.

Norris, A. [2006] "The isotropic material closest to a given anisotropic material," Journal of Mechanics of Materials and Structures 1(2), 223-238.

Rovati, M. and Taliercio, A. [2003] "Stationarity of the strain energy density for some classes of anisotropic solids," International Journal of Solids Structures 40(22), 6043-6075.

Rychlewski, J. [1995] "Unconventional approach to linear elasticity," Archives of Mechanics 52(2), 149-171.

Segurado, J. and Llorca, J. [2002] "A numerical approximation to the elastic properties of sphere-reinforced composites," Journal of the Mechanics and Physics of Solids 50(10), 2107-2121.

Ting, T. C. T. [1996] Anisotropic Elasticity: Theory and Applications (Oxford University Press, New York).

Ting, T. C. T. [2006] "On anisotropic elastic materials for which one sheet of the slowness surface is a sphere or a cross-section of a slowness sheet is a circle," Wave Motion 43(4), 287-300.

Ting, T. C. T. and He, Q. C. [2006] "Decomposition of elasticity tensors and tensors that are structurally invariant in three dimensions," The Quarterly Journal of Mechanics and Applied Mathematics 59(3), 323-341.

Truesdell, C. [1966] "Existence of longitudinal waves," The Journal of the Acoustical Society of America 40(3), 729-730.

Truesdell, C. [1968] "Comment on longitudinal waves," The Journal of the Acoustical Society of America 43(1), 170. 